\title{
Obstructive sleep apnoea as a cause of nocturnal nondipping blood pressure: recent evidence regarding clinical importance and underlying mechanisms
}

\author{
Sophie J. Crinion ${ }^{1,2}$, Silke Ryan ${ }^{1,2}$ and Walter T. McNicholas ${ }^{1,2}$ \\ Affiliations: ${ }^{1}$ Dept of Respiratory and Sleep Medicine, St Vincent's University Hospital, Dublin, Ireland. ${ }^{2}$ School \\ of Medicine and Medical Science, University College Dublin, Dublin, Ireland. \\ Correspondence: Walter McNicholas, Pulmonary and Sleep Disorders Unit, St Vincent's University Hospital, \\ Elm Park, Dublin 4, Ireland. E-mail: walter.mcnicholasQucd.ie
}

@ERSpublications

OSA is strongly associated with nondipping nocturnal blood pressure, which may help predict clinical significance http://ow.ly/HToK305OnCV

Cite this article as: Crinion SJ, Ryan S, McNicholas WT. Obstructive sleep apnoea as a cause of nocturnal nondipping blood pressure: recent evidence regarding clinical importance and underlying mechanisms. Eur Respir J 2017; 49: 1601818 [https://doi.org/10.1183/13993003.01818-2016].

Obstructive sleep apnoea (OSA) is highly prevalent, with recent general population studies indicating that up to $50 \%$ of males and $23 \%$ of females have moderate or severe sleep disordered breathing (SDB) based on an apnoea-hypopnoea index (AHI) of $>15$ events $\cdot \mathrm{h}^{-1}[1,2]$. OSA is recognised as an independent risk factor for systemic hypertension [3] and a nondipping nocturnal blood pressure profile is particularly likely in patients with OSA [4], even in the absence of significant hypertension. Furthermore, hypertension is increasingly recognised as an important predictor of prevalent OSA and appears to be more predictive than excessive day-time sleepiness in some settings [5]. However, recent evidence also indicates that only moderate-severe OSA (AHI $>20$ events $\cdot \mathrm{h}^{-1}$ ) constitutes a significant independent risk for hypertension [2] and cluster analysis of sleep clinic populations indicates that particular population subtypes are especially associated with hypertension [6].

These recent reports regarding the very high general prevalence of SDB and factors that influence the relationship with hypertension prompt a reassessment of the clinical relevance regarding the association between OSA and hypertension, especially nocturnal hypertension, and the underlying mechanisms that may contribute to the development of a nondipping nocturnal blood pressure profile in OSA patients. This topic is important in the context of the recent SAVE (Sleep Apnea Cardiovascular Endpoints) trial report [7] involving 2717 patients with established cardiovascular disease and moderate or severe OSA associated with minimal sleepiness who were randomised to best usual care with or without added continuous positive airway pressure (CPAP) therapy and followed for up to 7 years. CPAP therapy was not associated with any improvement in cardiac or cerebrovascular outcome, although data on secondary and other end-points in the report indicated a small but significant reduction in diastolic blood pressure. This finding is particularly relevant in the context that the average compliance with CPAP was only $3.3 \mathrm{~h}$ and a recent meta-analysis indicates that beneficial effects of CPAP on blood pressure in OSA patients with minimal sleepiness are largely confined to patients using CPAP for $>4 \mathrm{~h}$ per night [8].

Received: Sept 142016 | Accepted after revision: Oct 022016

Conflict of interest: None declared.

Copyright OERS 2017 
Hypertension is present in up to $50 \%$ of patients with OSA [3], which is close to double the prevalence of hypertension in general population studies [9]. Blood pressure normally follows a diurnal pattern such that the average nocturnal systolic blood pressure is $>10 \%$ lower than during day-time. Loss of this nocturnal dipping blood pressure pattern in both normotensive and hypertensive subjects is associated with a worse cardiovascular prognosis [10], which is particularly relevant in OSA because of the high likelihood of a nocturnal nondipping profile [3]. Data from the Wisconsin Sleep Cohort Study indicate a dose-response increase in the development of nondipping hypertension with severity of SDB at baseline when followed for 7 years [11], which was confirmed by a recent report demonstrating that in patients attending a cardiology clinic with known cardiovascular disease and moderate or severe OSA there is a $4 \%$ increase in the odds of having a nondipping blood pressure profile per unit increase in AHI [4]. Further recent data from the Wisconsin Cohort indicate that SDB during rapid eye movement sleep is particularly associated with a nondipping nocturnal blood pressure pattern [12].

In general terms, the presence of a nondipping nocturnal blood pressure profile is associated with an increased incidence of cardiovascular events regardless of the underlying absolute blood pressure values. In one normotensive cohort, a nondipping blood pressure pattern was reported in $36 \%$ of subjects and the adjusted hazards ratio of cardiovascular events in nondippers was 2.44 compared with dippers [10]. In another randomly selected population cohort, nondipping status also predicted a higher risk of cardiovascular disease [13]. The prevalence of a nondipping blood pressure profile has been reported as high as $53 \%$ in a treated hypertensive population [14] in whom its presence independently predicts cardiovascular events even after adjustment for 24-h blood pressure [15]. Furthermore, a recent meta-analysis supports an association in untreated hypertensive subjects between a nondipping pattern and an increased risk of left ventricular structural alterations [16]. Thus, the nocturnal dipping status represents an important element in risk stratification for cardiovascular comorbidity. Importantly, recent evidence confirms that cardiovascular events are more frequent in OSA patients with a nondipping blood pressure profile even in the absence of diagnosed hypertension [17].

The mechanisms by which OSA contribute to the presence of nondipping blood pressure and indeed systemic hypertension in general are likely multifactorial [3], and are summarised in figure 1. Key factors relating to OSA that promote the potential mechanisms of blood pressure elevations include intermittent hypoxia and recurring micro-arousals from sleep, both of which are central features of SDB events. Intermittent hypoxia has long been recognised as one of the mechanisms in the development of OSA-related hypertension, with early animal studies showing a robust association between intermittent hypoxia as a marker of OSA and both acute and persistent increases in blood pressure [18]. However, recent evidence indicates that fragmented sleep also represents a distinct trigger factor for elevated blood pressure in that sleep fragmentation and frequent arousals in patients with periodic leg movements have also been found to be associated with elevated blood pressure [19]. Even in children, periodic leg movements are frequently associated with micro-arousals from sleep and have been linked to the development of nocturnal hypertension [20].

Sympathetic excitation has long been recognised as an important feature of OSA, and sympathetic discharge measured by direct recording of sympathetic nerve activity in leg muscle is increased at the termination of apnoea and hypopnoea events [21] with associated transient elevation in blood pressure. Crucially, over time, these brief surges in sympathetic activity during sleep in OSA and associated nocturnal blood pressure rise

FIGURE 1 Schematic diagram of the proposed relationship between obstructive sleep apnoea (OSA) and systemic hypertension. RAAS: reninangiotensin-aldosterone system.

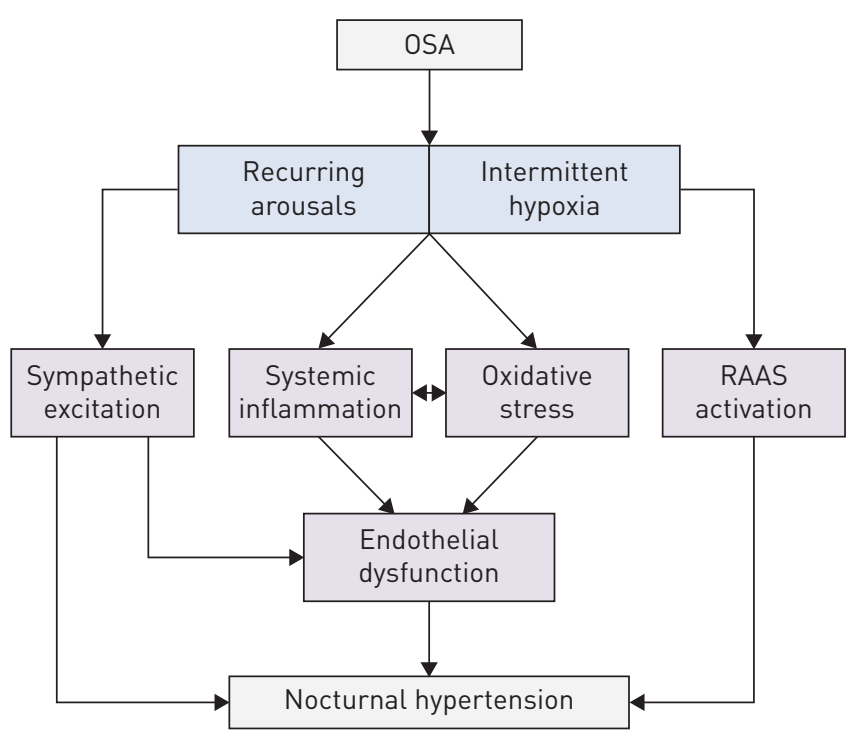


perpetuate beyond the offending events, with sustained sympathetic activity persisting into the day-time and associated elevation of blood pressure. Recent evidence indicates that both intermittent hypoxia [22] and recurring arousals [23] independently contribute to sympathetic excitation and associated blood pressure elevation, including studies of intermittent hypoxia in normal volunteers [24].

The renin-angiotensin-aldosterone system (RAAS) regulates blood pressure through the vasoactive peptide angiotensin II [25] and recent evidence indicates that RAAS activity is progressively increased with increasing severity of OSA [26], but reduced following CPAP therapy. Increased RAAS activity promotes the genesis of a nondipping blood pressure profile [27] and blocking the RAAS with an angiotensin II receptor blocker is associated with restoration of a nocturnal dipping blood pressure pattern [28]. Thus, RAAS activation in OSA is implicated in the development of a nondipping blood pressure profile as well as the propagation of a more sustained hypertensive state.

Systemic inflammation and oxidative stress are both evident in OSA, and have been linked to cardiovascular disease [29], particularly by promoting atherosclerosis. Recent findings of higher circulating levels of the inflammatory factor interleukin-2 in OSA patients with a nondipping blood pressure profile also support a role for inflammation in the development of nondipping blood pressure [30].

Endothelial dysfunction, a precursor of atherosclerosis [31] and predictor of incident cardiovascular events [32], is widely reported in patients with OSA [33]. Both intermittent hypoxia [34] and sleep fragmentation [35] contribute to endothelial dysfunction with intermediate pathways that include reduced bioavailability of the vasodilator substance endothelial nitric oxide, oxidative stress, systemic inflammation and increased sympathetic drive (figure 1). Nondipping nocturnal blood pressure is also associated with vascular endothelial dysfunction and reduced nitric oxide levels [36]. Furthermore, nocturnal nondippers demonstrate a reduced number of endothelial progenitor cells, which are responsible for repairing vascular damage and maintaining homeostasis [37], a finding also reported in patients with OSA [38].

The recent evidence that SDB is so very highly prevalent supports the view that additional factors such as related comorbidities should be considered in the diagnosis of a clinically significant disorder, particularly as traditional symptoms such as day-time sleepiness correlate so poorly with OSA severity as measured by AHI $[5,39]$. In this context, emerging evidence supports the view that hypertension and particularly the loss of nocturnal dipping blood pressure may represent a significant associated clinical factor that identifies patients with clinically significant OSA. Furthermore, as OSA, via intermittent hypoxia and recurring arousals, triggers a range of intermediate mechanism that promote nocturnal elevation of blood pressure, further investigation of these complex mechanisms in the progression of OSA patients from normotensive to hypertensive, and particularly the mechanisms contributing to nocturnal elevation of blood pressure, may provide important insights into the pathophysiology and consequences of hypertension in general. In practical terms, the highly prevalent association of OSA with elevated nocturnal blood pressure implies that 24 -h ambulatory blood pressure recordings should be a routine part of the assessment of patients with OSA, as recently recommended by a joint Task Force of the European Respiratory Society and the European Society of Hypertension [3].

\section{References}

1 Arnardottir ES, Bjornsdottir E, Olafsdottir KA, et al. Obstructive sleep apnoea in the general population: highly prevalent but minimal symptoms. Eur Respir J 2016; 47: 194-202.

2 Heinzer R, Vat S, Marques-Vidal P, et al. Prevalence of sleep-disordered breathing in the general population: the HypnoLaus study. Lancet Respir Med 2015; 3: 310-318.

3 Parati G, Lombardi C, Hedner J, et al. Recommendations for the management of patients with obstructive sleep apnoea and hypertension. Eur Respir J 2013; 41: 523-538.

4 Seif F, Patel SR, Walia HK, et al. Obstructive sleep apnea and diurnal nondipping hemodynamic indices in patients at increased cardiovascular risk. J Hypertens 2014; 32: 267-275.

5 Ustun B, Westover MB, Rudin C, et al. Clinical prediction models for sleep apnea: the importance of medical history over symptoms. J Clin Sleep Med 2016; 12: 161-168.

6 Bailly S, Destors M, Grillet Y, et al. Obstructive sleep apnea: a cluster analysis at time of diagnosis. PLoS One 2016; 11: e0157318.

7 McEvoy RD, Antic NA, Heeley E, et al. CPAP for prevention of cardiovascular events in obstructive sleep apnea. N Engl J Med 2016; 375: 919-931.

8 Bratton DJ, Stradling JR, Barbé F, et al. Effect of CPAP on blood pressure in patients with minimally symptomatic obstructive sleep apnoea: a meta-analysis using individual patient data from four randomised controlled trials. Thorax 2014; 69: 1128-1135.

9 Cutler JA, Sorlie PD, Wolz M, et al. Trends in hypertension prevalence, awareness, treatment, and control rates in United States adults between 1988-1994 and 1999-2004. Hypertension 2008; 52: 818-827.

10 Hermida RC, Ayala DE, Mojón A, et al. Blunted sleep-time relative blood pressure decline increases cardiovascular risk independent of blood pressure level - the "normotensive non-dipper" paradox. Chronobiol Int 2013; 30: 87-98.

11 Hla KM, Young T, Finn L, et al. Longitudinal association of sleep-disordered breathing and nondipping of nocturnal blood pressure in the Wisconsin Sleep Cohort study. Sleep 2008; 31: 795-800. 
12 Mokhlesi B, Hagen EW, Finn LA, et al. Obstructive sleep apnoea during REM sleep and incident non-dipping of nocturnal blood pressure: a longitudinal analysis of the Wisconsin Sleep Cohort. Thorax 2015; 70: 1062-1069.

13 Boggia J, Li Y, Thijs L, et al. Prognostic accuracy of day versus night ambulatory blood pressure: a cohort study. Lancet 2007; 370: 1219-1229.

14 de la Sierra A, Redon J, Banegas JR, et al. Prevalence and factors associated with circadian blood pressure patterns in hypertensive patients. Hypertension 2009; 53: 466-472.

15 Fagard RH, Thijs L, Staessen JA, et al. Night-day blood pressure ratio and dipping pattern as predictors of death and cardiovascular events in hypertension. J Hum Hypertens 2009; 23: 645-653.

16 Cuspidi C, Sala C, Tadic M, et al. Non-dipping pattern and subclinical cardiac damage in untreated hypertension: a systematic review and meta-analysis of echocardiographic studies. Am J Hypertens 2015; 28: 1392-1402.

17 Sasaki N, Ozono R, Edahiro Y, et al. Impact of non-dipping on cardiovascular outcomes in patients with obstructive sleep apnea syndrome. Clin Exp Hypertens 2015; 37: 449-453.

18 Fletcher EC. Physiological consequences of intermittent hypoxia: systemic blood pressure. J Appl Physiol 2001; 90: $1600-1605$

19 Koo BB, Sillau S, Dean DA, et al. Periodic limb movements during sleep and prevalent hypertension in the multi-ethnic study of atherosclerosis. Hypertension 2015; 65: 70-77.

20 Wing YK, Zhang J, Ho CKW, et al. Periodic limb movement during sleep is associated with nocturnal hypertension in children. Sleep 2010; 33: 759-765.

21 Somers VK, Dyken ME, Clary MP, et al. Sympathetic neural mechanisms in obstructive sleep apnea. J Clin Invest 1995; 96: 1897-1904.

22 Weiss JW, Tamisier R, Liu Y. Sympathoexcitation and arterial hypertension associated with obstructive sleep apnea and cyclic intermittent hypoxia. J Appl Physiol 2015; 119: 1449-1454.

23 Chouchou F, Pichot V, Pépin JL, et al. Sympathetic overactivity due to sleep fragmentation is associated with elevated diurnal systolic blood pressure in healthy elderly subjects: the PROOF-SYNAPSE study. Eur Heart J 2013; 34: $2122-2131$

24 Tamisier R, Pépin JL, Rémy J, et al. 14 nights of intermittent hypoxia elevate daytime blood pressure and sympathetic activity in healthy humans. Eur Respir J 2011; 37: 119-128.

25 te Riet L, van Esch JHM, Roks AJM, et al. Hypertension: renin-angiotensin-aldosterone system alterations. Circ Res 2015; 116: 960-975.

26 Nicholl DDM, Hanly PJ, Poulin MJ, et al. Evaluation of continuous positive airway pressure therapy on reninangiotensin system activity in obstructive sleep apnea. Am J Respir Crit Care Med 2014; 190: 572-580.

27 Fukuda M, Urushihara $M$, Wakamatsu $T$, et al. Proximal tubular angiotensinogen in renal biopsy suggests nondipper BP rhythm accompanied by enhanced tubular sodium reabsorption. J Hypertens 2012; 30: 1453-1459.

28 Fukuda M, Wakamatsu-Yamanaka T, Mizuno M, et al. Angiotensin receptor blockers shift the circadian rhythm of blood pressure by suppressing tubular sodium reabsorption. Am J Physiol Renal Physiol 2011; 301: F953-F957.

29 Ryan S, Taylor CT, McNicholas WT. Systemic inflammation: a key factor in the pathogenesis of cardiovascular complications in obstructive sleep apnoea syndrome? Thorax 2009; 64: 631-636.

30 Sarinc Ulasli S, Sarraydın M, Gunay E, et al. Effects of nondipping pattern on systemic inflammation in obstructive sleep apnea. Sleep Breath 2015; 19: 1185-1190.

31 Gimbrone MA, García-Cardeña G. Endothelial cell dysfunction and the pathobiology of atherosclerosis. Circ Res 2016; 118: 620-636.

32 Yeboah J, Folsom AR, Burke GL, et al. Predictive value of brachial flow-mediated dilation for incident cardiovascular events in a population-based study: the multi-ethnic study of atherosclerosis. Circulation 2009; 120: 502-509.

33 Wang J, Yu W, Gao M, et al. Impact of obstructive sleep apnea syndrome on endothelial function, arterial stiffening, and serum inflammatory markers: an updated meta-analysis and metaregression of 18 studies. $\mathrm{J} \mathrm{Am}$ Heart Assoc 2015; 4: e002454.

34 Hoyos CM, Melehan KL, Liu PY, et al. Does obstructive sleep apnea cause endothelial dysfunction? A critical review of the literature. Sleep Med Rev 2015; 20: 15-26.

35 Carreras A, Zhang SX, Peris E, et al. Chronic sleep fragmentation induces endothelial dysfunction and structural vascular changes in mice. Sleep 2014; 37: 1817-1824.

36 Lee KW, Blann AD, Lip GYH. High pulse pressure and nondipping circadian blood pressure in patients with coronary artery disease: relationship to thrombogenesis and endothelial damage/dysfunction. Am J Hypertens 2005; 18: 104-115.

37 Kim S, Kim NH, Kim YK, et al. The number of endothelial progenitor cells is decreased in patients with non-dipper hypertension. Korean Circ J 2012; 42: 329-334.

38 de la Pena M, Barcelo A, Barbe F, et al. Endothelial function and circulating endothelial progenitor cells in patients with sleep apnea syndrome. Respiration 2008; 76: 28-32.

39 Marti-Soler H, Hirotsu C, Marques-Vidal P, et al. The NoSAS score for screening of sleep-disordered breathing: a derivation and validation study. Lancet Respir Med 2016; 4: 742-748. 